\title{
The BUD2 mutation affects plant architecture through altering cytokinin and auxin responses in Arabidopsis
}

$\mathrm{Xia} \mathrm{Cui}^{1,{ }^{*}}$, Chunmin $\mathrm{Ge}^{1,3,{ }^{*} \text {, Renxiao Wang }}{ }^{1,4}$, Huanzhong Wang ${ }^{1,5}$, Weiqi Chen ${ }^{2}$, Zhiming Fu ${ }^{1}$, Xiangning Jiang ${ }^{2}$, Jiayang $\mathrm{Li}^{1}$, Yonghong Wang ${ }^{1}$

${ }^{I}$ State Key Laboratory of Plant Genomics and National Center for Plant Gene Research, Institute of Genetics and Developmental Biology, Chinese Academy of Sciences, Beijing 100101, China; ${ }^{2}$ The Tree and Ornamental Plant Breeding and Biotechnology Laboratory of State Forestry Administration, College of Life Sciences and Biotechnology, Beijing Forestry University, Beijing 100083, China

The ratio of auxin and cytokinin plays a crucial role in regulating aerial architecture by promoting or repressing axillary bud outgrowth. We have previously identified an Arabidopsis mutant bud2 that displays altered root and shoot architecture, which results from the loss-of-function of $S$-adenosylmethionine decarboxylase 4 (SAMDC4). In this study, we demonstrate that BUD2 could be induced by auxin, and the induction is dependent on auxin signaling. The mutation of BUD2 results in hyposensitivity to auxin and hypersensitivity to cytokinin, which is confirmed by callus induction assays. Our study suggests that polyamines may play their roles in regulating the plant architecture through affecting the homeostasis of cytokinins and sensitivities to auxin and cytokinin.

Keywords: $B U D 2$, shoot branching, auxin, cytokinin

Cell Research (2010) 20:576-586. doi:10.1038/cr.2010.51; published online 13 April 2010

\section{Introduction}

Polyamines, including diamine putrescine, triamine spermidine and tetraamine spermine, are low-molecularmass organic cations. They exist widely in all living organisms and are essential for their survival, because blocking of the biosynthesis of polyamines leads to lethal phenotypes in animals [1] and higher plants [2-4]. In higher plants, polyamines have been proposed to function in response to environmental stresses and in regulating growth and development [4-11]. Plant molecular and physiological studies over past decades have shown that higher plants defective in producing polyamines often have altered plant architecture, with reduced plant

\footnotetext{
*These two authors contributed equally to this work.

Correspondence: Yonghong Wang

Tel: +86-10-64806008; Fax: +86-10-64883428

E-mail: yhwang@genetics.ac.cn

${ }^{3}$ Current address: The Stowers Institute for Medical Research, Kansas, USA. ${ }^{4}$ Current address: Institute of Biology II, Albert Ludwigs University, Freiburg, Germany. ${ }^{5}$ Current address: The Samuel Roberts Noble Foundation, Ardmore, USA.

Received 1 September 2009; revised 30 November 2009; accepted 26 January 2010, published online 13 April 2010
}

height or more branches in shoots and roots [4, 10-13]. However, the underlying mechanisms still remain largely elusive.

Branches of higher plants originate from the axillary meristems (AMs) of shoots, and formation of a branch generally consists of the initiation of a new AM and its subsequent outgrowth. However, an AM may arrest its growth under some conditions, forming a dormant bud. The dormant buds will release their outgrowth once they sense a permissible environmental or developmental signal. In many plant species, axillary buds become dormant due to the inhibiting effects of the primary shoot apex on the outgrowth of AMs, a phenomenon known as 'apical dominance'. Auxin was first regarded as a direct regulator in this process [14], a notion strengthened thereafter by physiological studies on decapitated shoot apices [1517], and by analyzing auxin biosynthesis, transport and signaling [18-29] in plants. However, when radiolabelled auxin was applied to a decapitated stump, the outgrowth of axillary buds was inhibited even though radiolabelled auxin was not found to accumulate in axillary buds, suggesting an indirect suppression effect of auxin on the AM outgrowth $[28,30]$ and the presence of second messengers. 
Cytokinin has been proposed as a second messenger that mediates the action of auxin in controlling the apical dominance, because it promotes the outgrowth of lateral buds when directly applied to buds [31]. Although an antagonistic role of auxin and cytokinin in the regulation of apical dominance has been postulated for decades, little is known about the underlying molecular mechanisms. Recent studies have shown that auxin can regulate the expressions of IPT genes and that cytokinin biosynthesis is directly affected by auxin in local shoots [32, 33], suggesting that auxin may modulate cytokinin concentration and thus represses AM outgrowth [31, 34-36].

Recent studies on a series of branching mutants, such as more axillary growth (max) of Arabidopsis [37-40], ramosus (rms) mutants of pea [41-43], decreased apical dominance (dad) mutants of petunia [44, 45] and dwarf (d) mutants of rice [46-51], have revealed strigolactone as a second messenger of auxin action on the control of AM outgrowth [52, 53]. Strigolactones, a group of terpenoid lactones that have been found in root exudates of diverse plant species, are synthesized from carotenoids in roots and transported acropetally or synthesized locally to repress the outgrowth of shoot branches [38, 54-56]. However, the biosynthesis and signaling pathways still remain to be elucidated.

Moreover, polyamines have been demonstrated to play a regulatory role in controlling the outgrowth of axillary buds in the Arabidopsis mutant, bushy and dwarf 2 (bud2) [4], which shows a dwarf and bushy phenotype. The $B U D 2$ gene encodes $S$-adenosylmethionine decarboxylase 4 (SAMDC4), an enzyme required for the biosynthesis of polyamines in Arabidopsis thaliana. In this study, we show that polyamines may play their roles in regulating the plant architecture through affecting the homeostasis of cytokinins and sensitivities to auxin and cytokinin.

\section{Results}

The bud2 plant shows altered hypocotyl elongation and lateral bud outgrowth

Our previous study showed that the bushy and dwarf phenotype of the Arabidopsis bud2 mutant plant results from the defect in the biosynthesis of polyamines [4]. To investigate whether the loss of apical dominance of bud2 plants involves auxin action, we examined the hypocotyl elongation at higher temperature and the lateral shoot development after decapitation of wild-type and bud2 mutant plants $[43,57,58]$. Identification of temperatureinduced hypocotyl elongation is a simple and rapid assay in the study of an auxin-mediated growth response [59]. As shown in Figure 1A, the hypocotyl length of the
A

A $\quad 20^{\circ} \mathrm{C}$

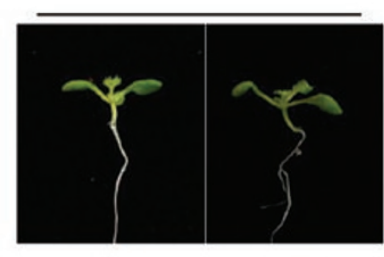

WT

bud2-2

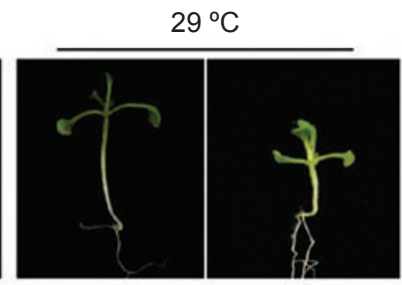

WT

bud2-2

B

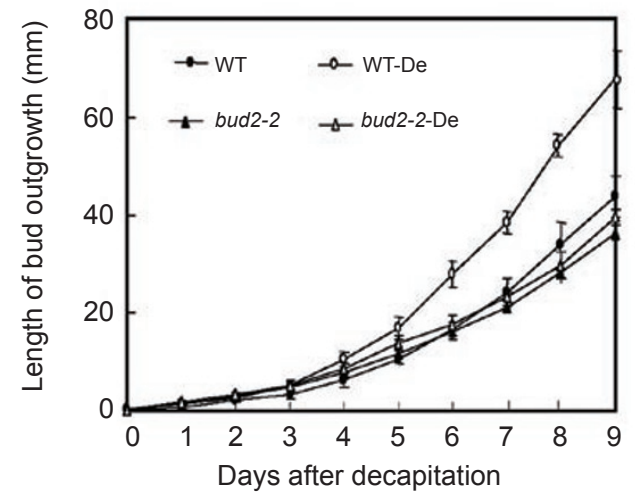

Figure 1 Hypocotyl elongation at high temperature and branching of decapitated plants. (A) Induction of hypocotyl elongation by high temperature. Wild-type and bud 2 mutant seedlings were grown on $1 / 2 \mathrm{MS}$ plates at $20^{\circ} \mathrm{C}$ or $29^{\circ} \mathrm{C}$ for 9 days and photographed at the same magnification. (B) The growth rate of the lowest cauline bud in intact and decapitated plants. The lowest cauline bud length of the intact and decapitated plants (decapitated above the lowest cauline bud $1.0 \mathrm{~cm}$ ) were measured every $24 \mathrm{~h}$ until day 9 . Error bars show SE $(n=5)$.

9-day-old bud2 seedlings grown at $29{ }^{\circ} \mathrm{C}$ was seriously inhibited compared with that of the wild-type seedlings, suggesting that bud2 may have an altered auxin-dependent developmental process. This was further strengthened by the decapitation experiment. As shown in Figure $1 \mathrm{~B}$, the outgrowth rate of lateral buds of decapitated wild-type plants was significantly accelerated compared with that of the decapitated bud2 plants, indicating that the $b u d 2$ plant has an abnormal response to shoot decapitation. These results strongly suggest that the bud2 mutant plant has an altered auxin-related development.

\section{The bud2 plant is hyposensitive to exogenous auxin}

To determine whether the biosynthesis, transport or signaling of auxin is altered in the bud2 mutant plant, we compared the IAA concentration, polar auxin transport (PAT) and response to auxin between the wildtype and mutant plants. No significant differences in the IAA contents of seedlings and the PAT capacity of 
roots and inflorescence stems were found between bud2 and wild-type plants (see Supplementary information, Figures S1 and S2). However, application of a low concentration of exogenous auxin (1.0 nM 1-naphthalene acetic acid, NAA) to bud2 seedlings showed no effect on lateral root formation, in contrast to wild-type plants (Figure 2A), indicating that the bud2 plant is relatively insensitive to exogenous auxin in promoting lateral root formation. Furthermore, we assayed the auxin effect on the axillary bud outgrowth using a split plate procedure reported previously [22]. As shown in Figure 2B, the exogenous NAA almost completely inhibited the axillary bud outgrowth of the wild-type plants, but it showed no significant impeding effect on bud2 and axr1-3, a mutant defective in the auxin-signaling pathway [60]. These results demonstrate that the lateral root formation and axillary bud elongation of the bud2 plants are hyposensitive to exogenous auxin, suggesting that polyamines may be involved in regulating root and shoot branching through affecting auxin response in Arabidopsis. Consistently, we found that the expression levels of primary auxin-responsive genes, IAAI and IAA4 [61], in the bud2-2 plant were significantly attenuated after $60 \mathrm{~min}$ of IAA treatment (Figure 2C). Taken together, these results indicate that auxin signaling is defective in bud2 mutant plants.

\section{Induction of BUD2 by auxin}

To determine whether $B U D 2$ expression is regulated by auxin or cytokinin, we determined the BUD2 expression levels in wild-type seedlings on auxin and cytokinin treatments. RNA gel blot analysis revealed that the $B U D 2$ transcripts were obviously accumulated at $30 \mathrm{~min}$ after IAA treatment and reached a maximal level at 120 min, while no significant alteration was observed after
A

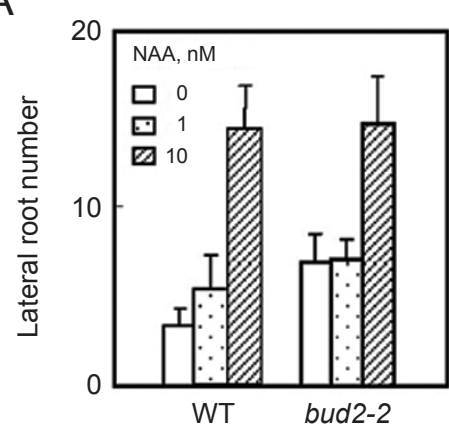

C

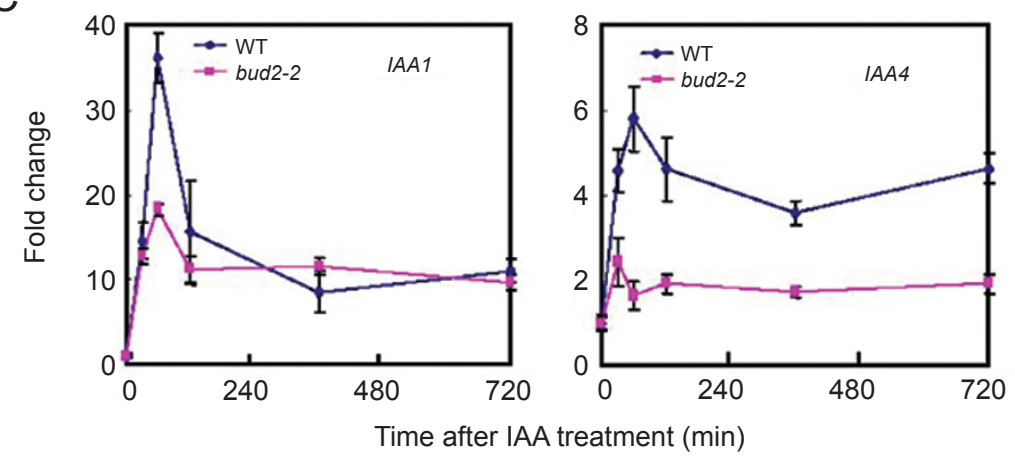

B

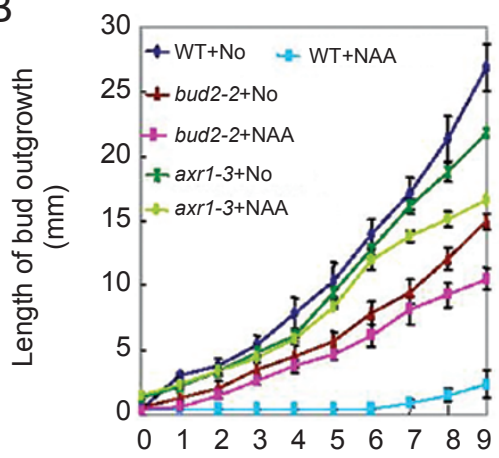

Figure 2 Response of the bud2 plant to exogenous auxin. (A) The lateral root formation of bud2-2 mutant seedlings is less sensitive to exogenous $\alpha$-NAA. The light-grown 3-day-old seedlings were transferred from the $1 / 2$ MS plate onto the plate containing different concentrations of $\alpha$-NAA. After 4 days of treatment, the average number of visible lateral roots was measured for each genotype. Experiments were repeated twice. Error bars show SE $(n=20)$. (B) Effect of $\alpha$-NAA on lateral inflorescence outgrowth from excised nodes of the wild-type, bud2-2 and axr1-3 plants. The lowermost cauline node was excised from the elongating primary inflorescence of axenically grown plants and inserted between two separate agar slabs. Slabs contained either no $\alpha$-NAA or $1.0 \mu \mathrm{M} \alpha$-NAA in contact with the apical or basal part of the stem. Lateral shoot lengths (mean \pm SE) were determined every $24 \mathrm{~h}$ until day 9 after excision. (C) Expression levels of IAA1 and IAA4 in wild-type and bud2-2 plants on IAA $(10 \mu \mathrm{M})$ treatment. Data represents the expression alteration of hormone-treated samples from three independent experiments. Bars represent \pm SD. 
treatment with 6-BA (Figure 3A). We also examined the expression levels of other SAMDC genes of Arabidopsis [4] upon auxin treatment. The result showed that
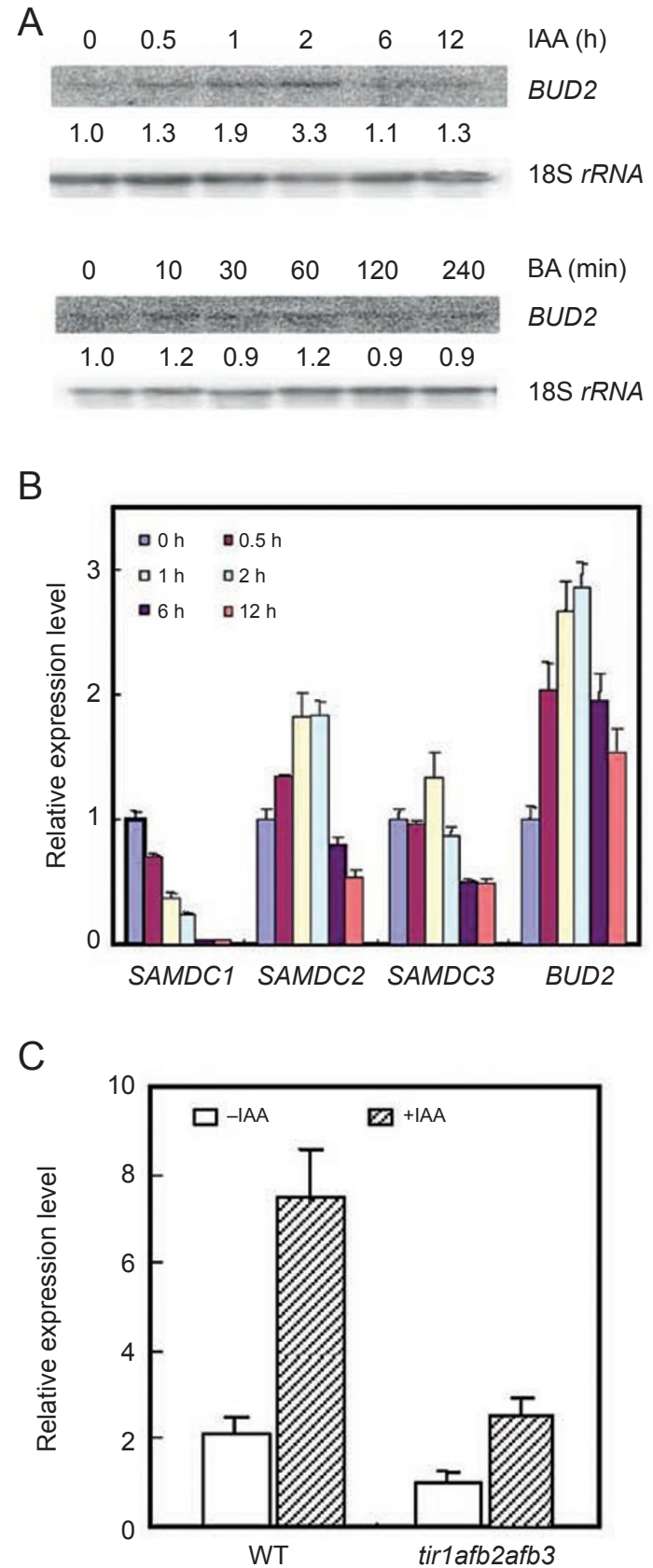

Figure 3 Induction of BUD2 by auxin. (A) Expression of BUD2 induced by auxin or cytokinin, showing the RNA gel blot analysis of the BUD2 expression levels in 10-day-old seedlings treated with IAA $(10 \mu \mathrm{M})$ or $6-\mathrm{BA}(10 \mu \mathrm{M})$. (B) Expression of SAMDCs revealed by $Q-P C R$ in 10-day-old seedlings treated with IAA (10 $\mu \mathrm{M})$. Bar represents \pm SD. (C) Expression of BUD2 in wild-type and tir1afb2afb3 plants treated with IAA $(10 \mu \mathrm{M})$ for $2 \mathrm{~h}$. Bars represent \pm SD.
$S A M D C 2$ and $S A M D C 3$ could be induced by auxin and had a similar tendency as the BUD2 response. However, $S A M D C 1$ transcript was strongly repressed with exogenous auxin treatment (Figure 3B). This result suggests that $S A M D C$ genes may interact with auxin signaling.

To determine whether BUD2 is induced specifically by auxin, we analyzed the expression level of $B U D 2$ in the auxin receptor tirlafb2afb3 triple mutant [62] by a quantitative PCR analysis. As shown in Figure 3C, the expression level of BUD2 increased by 2.5 -fold on IAA treatment in the wild-type plants, but by only $\sim 1.2$-fold in the tirlafb2afb3 triple mutant plants, suggesting that $B U D 2$ can be specifically induced by auxin.

Altered biosynthesis of cytokinins in bud2 plants

The outgrowth phenotype of the bud2 axillary buds may also result from altered cytokinin biosynthesis and/ or response, because cytokinin has long been proposed as a secondary messenger of IAA in controlling shoot branching [63-65]. We therefore compared the endogenous levels of various cytokinins between bud2-2 and wild-type seedlings. As shown in Table 1, the homeostasis of various endogenous cytokinins in the bud2 plant

Table 1 Cytokinin contents in bud2 and wild-type plants

\begin{tabular}{|c|c|c|c|}
\hline & WT & bud2-2 & WT $(\%)$ \\
\hline & Content $\left(\mathrm{pg} \mathrm{g}^{-1} \mathrm{FW}\right)$ & Content ( $\left.\mathrm{pg} \mathrm{g}^{-1} \mathrm{FW}\right)$ & \\
\hline$\overline{\mathrm{Z7G}}$ & $1043.87 \pm 63.18$ & $1477.66 \pm 21.21^{\mathrm{a}}$ & 141.6 \\
\hline Z9G & $547.52 \pm 35.56$ & $826.87 \pm 51.21^{\mathrm{a}}$ & 151.0 \\
\hline ZOG & $859.74 \pm 59.47$ & $1303.82 \pm 38.40^{\mathrm{a}}$ & 151.7 \\
\hline DZOG & $10.06 \pm 2.88$ & $7.23 \pm 1.50$ & 71.9 \\
\hline ZROG & $766.16 \pm 39.78$ & $942.90 \pm 38.75^{\mathrm{a}}$ & 123.1 \\
\hline iP7G & $2059.41 \pm 47.34$ & $1903.96 \pm 71.89^{\mathrm{a}}$ & 92.5 \\
\hline iP9G & $70.96 \pm 2.97$ & $73.13 \pm 9.56$ & 103.1 \\
\hline $\mathrm{tZ}$ & $28.74 \pm 3.27$ & $23.05 \pm 3.02$ & 80.2 \\
\hline tZR & $209.06 \pm 9.97$ & $244.50 \pm 7.53$ & 117.0 \\
\hline tZRMP & $424.79 \pm 15.71$ & $464.63 \pm 23.78$ & 109.4 \\
\hline DZR & $9.03 \pm 0.39$ & $12.13 \pm 1.63$ & 134.2 \\
\hline DZRMP & $97.58 \pm 4.99$ & $99.10 \pm 15.48$ & 101.6 \\
\hline iP & $14.92 \pm 1.42$ & $25.62 \pm 3.59^{b}$ & 171.7 \\
\hline iPR & $38.49 \pm 2.70$ & $49.31 \pm 3.58^{\mathrm{a}}$ & 128.1 \\
\hline iPRMP & $925.99 \pm 54.64$ & $788.84 \pm 25.08^{b}$ & 85.2 \\
\hline
\end{tabular}

${ }^{\mathrm{a}}$ Data represents means \pm SE from three independent samples.

${ }^{\mathrm{b}}$ Indicates the significant difference compared to wild type plants by Student's $t$-test. ${ }^{\mathrm{a}} P \leq 0.01,{ }^{\mathrm{b}} P \leq 0.05$. The statistical tests were performed using the SPSS v10.0. 

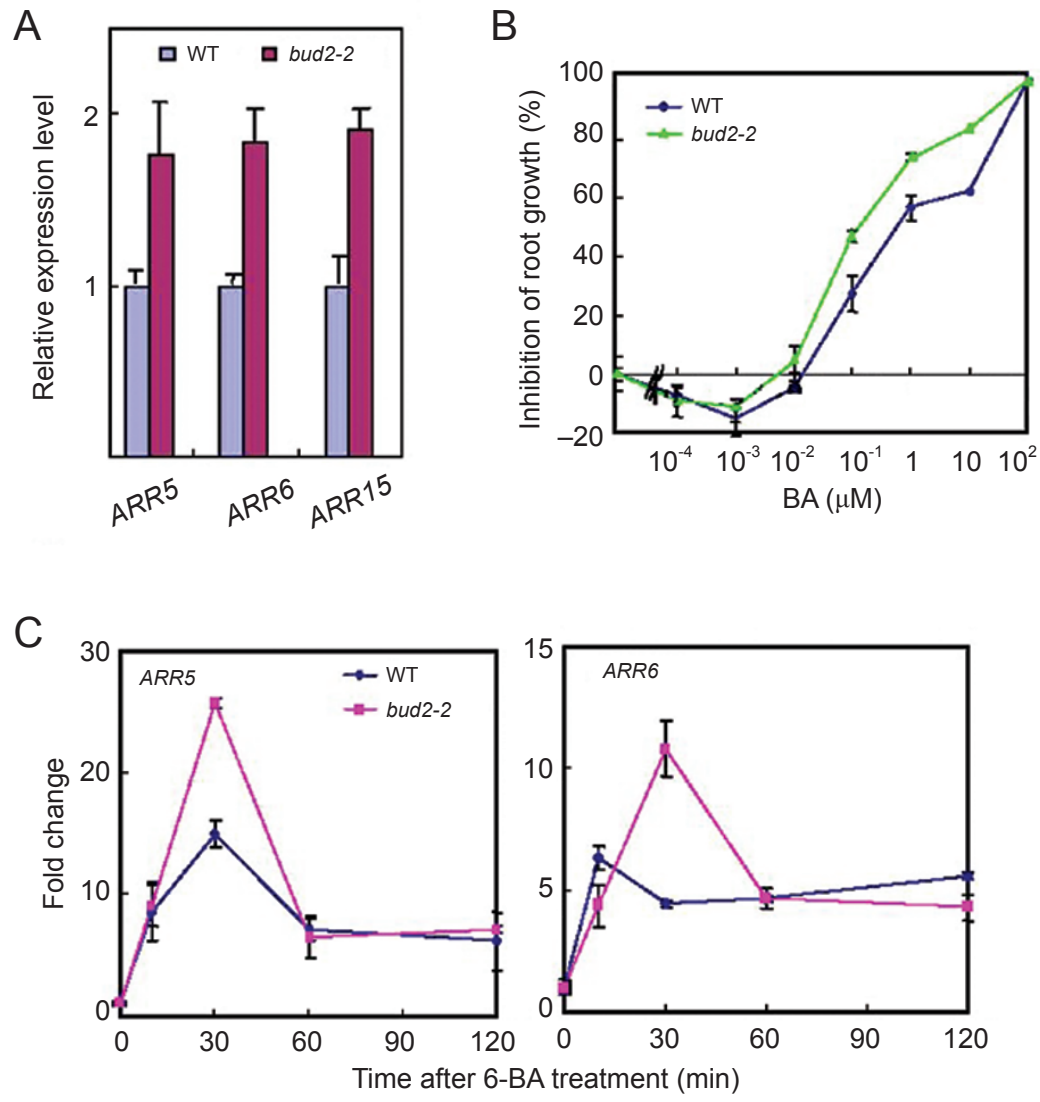

Figure 4 Response of bud2 to exogenous cytokinin. (A) Expression levels of $A R R 5, A R R 6$ and ARR15 of wild-type and bud2 seedlings at 25 DAG. Data represents the relative expression levels of these genes of three independent experiments. Bars represent \pm SD. (B) Comparison of the root elongation between wild-type and bud2-2 plants on 6-BA treatment. Data represents means $\pm \mathrm{SE}(n=30)$. (C) Expression of ARR5 and ARR6 in the aerial parts of wild-type and bud2-2 plants treated with 6 -BA $(10 \mu \mathrm{M})$. Data represents the alteration of the expression of hormone-treated samples from three independent experiments. Bars represent \pm SD.

was altered: trans-zeatin $O$-glucoside (ZOG), transzeatin 7-glucoside (Z7G), trans-zeatin 9-glucoside (Z9G), trans-zeatin riboside $O$-glucoside (ZROG), N6-(D2isopentenyl) adenosine (iPR) and N6-(D2-isopentenyl) adenine (iP) were significantly increased, but N6-(D2isopentenyl) adenine 7-glucoside (iP7G) and N6-(D2isopentenyl) adenosine monophosphate (iPRMP) were apparently decreased. These results demonstrate that the altered polyamine biosynthesis that resulted from the null mutation of BUD2 is able to cause an abnormal homeostasis of endogenous cytokinins, which may in turn affect the expression of cytokinin-responsive genes. We therefore examined the expression of cytokinin primary responsive genes, ARR5, ARR6 and ARR15, in the aerial part of 25-day-old plants [66-68]. The results showed that the expression levels of these cytokinin-responsive genes were significantly elevated in the bud2 mutant plant compared with the wild-type plant (Figure 4A).
The bud2 plant is hypersensitive to exogenous cytokinin

To further investigate whether BUD2 is involved in cytokinin signaling, we examined the sensitivity of bud2 and wild-type plants on cytokinin treatments. We measured the root elongation of $b u d 2$ and wild-type seedlings at the presence of various levels of exogenous cytokinin 6-BA. Compared to that of the wild-type plants, the inhibition of root elongation by 6-BA in bud2-2 seedlings took place at a much lower concentration, indicating that bud2 plants are hypersensitive to cytokinin (Figure 4B).

We further monitored the expression of two cytokinin primary responsive genes, $A R R 5$ and $A R R 6$, in wild-type and bud2-2 seedlings treated with 6-BA [69]. As shown in Figure 4C, the expression of ARR 5 and ARR6 was rapidly induced on the treatment of 6-BA and declined in both the wild-type and bud2-2 after 30-min treatment of 6-BA, but their induction levels in bud2-2 were much higher than those in the wild-type plants (Figure 4C). 
These results indicate that the response of bud2 to cytokinin is also changed.

\section{Genetic analysis of bud2 double mutant}

To further investigate the function of BUD2 in regulating axillary bud outgrowth, we generated a bud2-2 axr 1-3 double mutant (Figure 5). The bud2-2 axr1-3 double mutant showed an extremely dwarf and highly branched shoot phenotype; the length of lateral buds was much longer than the primary inflorescence and sometimes two lateral branches could be developed from a leaf axil (Figure 5D and 5E). Moreover, the high-order branches could also grow out from the axils of the firstand second-order branches in the double mutant (Figure $5 \mathrm{E})$.

Furthermore, the expression levels of ARR5 and ARR6 in the axillary buds $(<1.5 \mathrm{~mm}$ in length) were enhanced in the double mutant compared to either parental mutants (Figure 5F). These results suggest that BUD2 is likely involved in an IAA/cytokinin-regulated shoot branching pathway that is independent of AXR1.

Polyamines affect callus growth in response to the cytokinin/auxin ratio

Cytokinins are able to promote cell division and produce callus in concert with auxin in cultured plant tissues [70]. We further compared the callus production of wildtype and bud2 hypocotyl explants with various combinations of cytokinin zeatin and auxin 2,4-D. As shown in Figure 6, a significant difference in callus growth was observed at different combinations of auxin and cytokinin concentrations. The calli generated from the bud2 mutant hypocotyl explants grew much faster than those from the wild type at all the tested combinations of
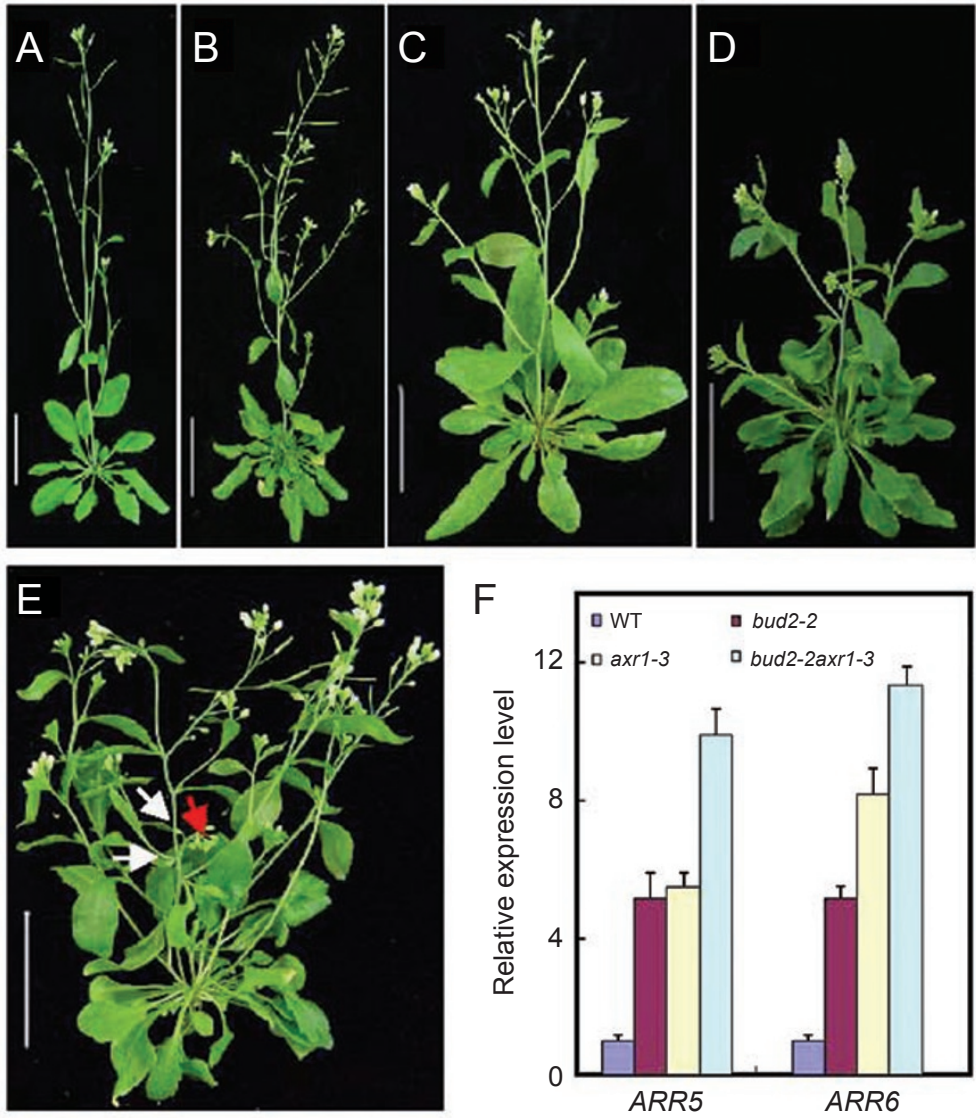

Figure 5 Phenotypes of the bud2-2axr1-3 double mutant. The phenotypes of wild type (A), axr1-3 (B), bud2-2 (C) and axr13bud2-2 (D) plants were photographed at day 10 after bolting. (E) The phenotype of bud2-2axr1-3 at day 18 after bolting. White arrows indicate two lateral buds in a leaf axil and the red arrow indicates the main shoot apex. Bars $=2 \mathrm{~cm}$. $(\mathbf{F})$ The expression of two cytokinin primary responsive genes, $A R R 5$ and $A R R 6$, in the lateral buds of wild-type, bud2-2, axr1-3 and axr1-3bud2-2 plants (bud length $<1.5 \mathrm{~mm}$ ). Data represents the relative expression levels of these genes in three independent experiments. Bars represent \pm SD. 


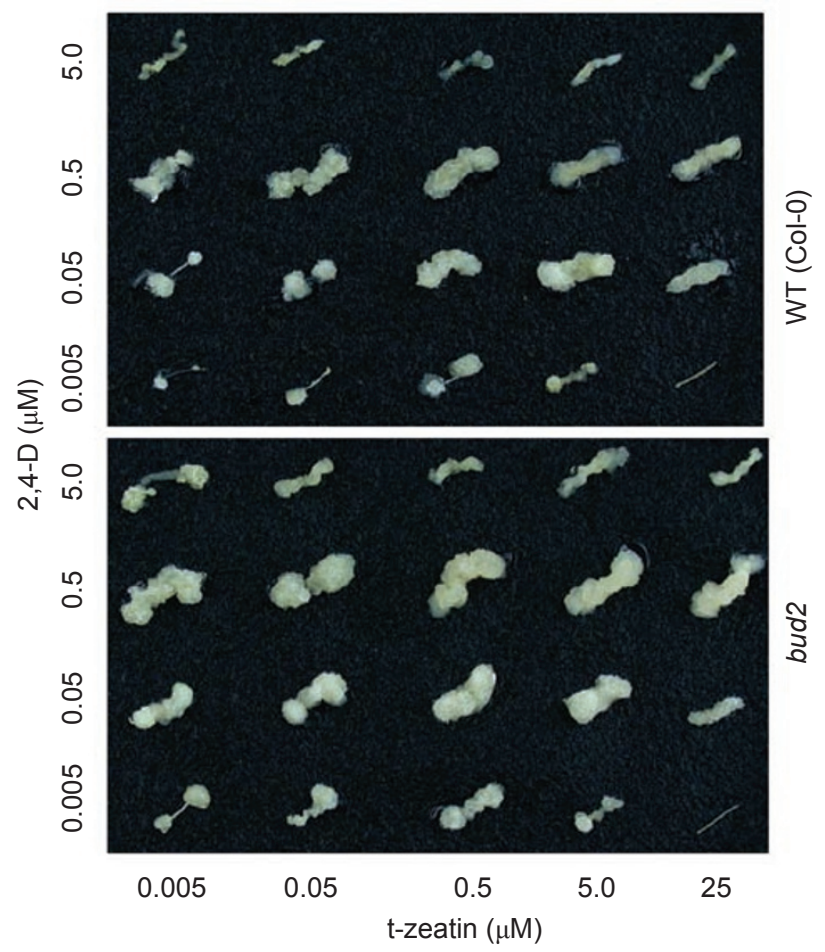

Figure 6 Callus induction of wild-type and bud2-1 explants. The hypocotyl explants from 5-day-old dark-grown seedlings of wild type (upper panel) and bud2-1 (lower panel) were cultured on GM plates containing the indicated concentrations of transzeatin and 2,4-D for 30 days in the dark. One representative callus from each treatment was collected and photographed.

trans-zeatin and 2,4-D, especially at the combination of lower concentration of cytokinin $(0.005 \mu \mathrm{M})$ and higher concentration of 2,4-D $(0.5 \mu \mathrm{M})$. This result is consistent with the seedling responses to exogenous cytokinin and auxin (Figures 2 and 4), indicating that the alteration of polyamines in bud2 results in an altered response to auxin and cytokinin.

\section{Discussion}

We have previously demonstrated that $B U D 2$ is the $S A M D C 4$ gene, which encodes an SAMDC. In the bud2 mutant plant, the homeostasis of polyamines is altered, leading to an obvious increase in putrescine and decreases in spermidine and spermine, which in turn leads to an altered plant morphology [4]. In recent years, studies on the biosynthesis of polyamines have indicated that polyamines are involved in a variety of plant developmental processes $[2,5-8,11,71,72]$, especially the formation of plant architecture including shoot apical dominance [58]. However, the mechanism underlying polyamine action needs to be elucidated. In this paper, we provide evidence that polyamines play an important role in controlling the branching of Arabidopsis through affecting the homeostasis of cytokinins and sensitivities to auxin and cytokinin.

Quantitative analysis of cytokinins demonstrated that the homeostasis of cytokinins is significantly altered in bud2 mutant plants (Table 1). The contents of 8 derivatives out of 15 examined cytokinins have significantly changed, among which 6 derivatives are increased. These elevated endogenous cytokinins, especially the active form iP, may explain why the expression levels of three cytokinin-inducible markers, ARR5, ARR6 and ARR15, are significantly enhanced in the bud2 mutant plant. This may also explain why the deficiency in polyamine biosynthesis promotes axillary bud outgrowth and causes a bushy phenotype.

Our study shows that deficiency in polyamine biosynthesis causes hyposensitivity to auxin. Compared with the wild-type plant, the bud2 mutant plant showed hyposensitivity to exogenous auxin NAA in lateral root formation or axillary bud outgrowth assays, which is reminiscent of previous findings in auxin-signaling mutants, $n p h 4$, arf19 or axr1-3 [22, 73]. Moreover, like most auxin-insensitive mutants [74], the expression levels of auxin-inducible genes are reduced in bud2. These findings strongly suggest that the polyamine contents may play an unidentified role in regulating the response to auxin. This hypothesis is further supported by the findings that $B U D 2$ is inducible by auxin and that the induction by auxin depends on a normal auxin-signaling pathway. In addition to $S A M D C s$, some other genes in the polyamine biosynthetic pathway, for example, $A C L 5$ and $P A O$, are also regulated by auxin $[8,75]$, supporting that polyamines are likely involved in the regulation of plant development by auxin.

Our results also demonstrate that $b u d 2$ is hypersensitive to cytokinin. As shown in the root growth inhibition assay, bud2 seedlings showed hypersensitivity to exogenous cytokinin 6-BA. Taken together, our results showed that the deficiency in polyamine biosynthesis led to hyposensitivity to auxin and hypersensitivity to cytokinin. This suggests that polyamines may play a role in regulating plant morphology by affecting plant response to the ratio of auxin and cytokinin. In fact, this is further confirmed by the callus production experiments. However, a challenge in the future is to understand how polyamines affect the sensitivities of plants to auxins and cytokinins. In addition, a recent study revealed that thermospermine, a structural isomer of spermine, can partially rescue acl5 mutant phenotype [13]. Further studies will explain whether thermospermine is also involved in 
BUD2-regulated stem elongation.

Taken together, our results suggest that polyamines play an important role in regulating plant architecture in Arabidopsis and function through the actions of auxin and cytokinin. We propose that polyamines may increase the sensitivity of auxin perception, and thus repress cytokinin biosynthesis or signaling.

\section{Materials and Methods}

\section{Plant growth}

A. thaliana ecotype Columbia-0 (Col-0) wild-type and mutant plants were grown on vermiculite saturated with $0.3 \times$ B5 medium under long photoperiod $\left(80-120 \mu \mathrm{Em}^{-2} \mathrm{sec}^{-1}\right)$ at $23^{\circ} \mathrm{C}$, as described previously [76]. For root observation, seeds were surface sterilized and vernalized at $4{ }^{\circ} \mathrm{C}$ for 4 days and seedlings were grown vertically at $23{ }^{\circ} \mathrm{C}$.

\section{RNA gel blot analysis, quantitative PCR}

Total RNA was isolated with the TRIzol reagent (Invitrogen, USA) and the RNA gel blot analysis was performed as previously described [77]. RNA (20 $\mu$ g per lane) was separated in a $0.8 \%$ agarose gel containing $10 \%$ formaldehyde, blotted onto a Hybond $\mathrm{N}^{+}$ membrane (Amersham) and probed with the PCR-amplified DNA fragments using a specific primer pair of SAMDC4 [4]. For the quantitative PCR analysis, $2.5 \mu \mathrm{g}$ of total RNA was treated with DNaseI (Gibco BRL) and then the first strand cDNA was synthesized using a cDNA synthesis kit (Promega, USA). The real-time PCR was performed with suitable procedures using the SYBR GREEN PCR Master Mix kit (ABI, USA) with specific primers, as described in Supplementary information, Table S1. All manipulations were carried out according to the instructions of manufacturers.

\section{Hormone response assays}

To test the sensitivity of the lateral root formation on auxin treatment, wild-type and bud2 seeds were grown vertically on $0.5 \times$ MS agar plates for 3 days after germination under continuous white light. The seedlings were transferred to plates containing various concentrations of NAA. The positions of the primary root tips were marked. Lateral roots were scored under a dissecting microscope and were accounted if visible primordia had formed. The axillary bud growth on auxin treatment in bud2-2 plants was assayed as described [22]. Seedlings of wild type, bud2-2 and axr1-3 were grown on $0.5 \times$ MS agar plates, and primary inflorescences appeared 25-30 days after germination. Inflorescence sections with the first cauline node and the axillary bud $(<0.5 \mathrm{~mm})$ were excised and placed over the gap between the two medium blocks in a split plate. The apical ends of the sections were inserted into the medium blocks with $1.0 \mu \mathrm{M}$ NAA. The split plates were held at $10^{\circ}$ off vertically with the node being upright. Growth of the lateral buds was then measured every $24 \mathrm{~h}$.

For the assay of the root elongation on cytokinin treatment, the wild-type and bud2 seedlings were grown vertically on $0.5 \times \mathrm{MS}$ agar plates containing various concentrations of 6-BA for 8 days under light and the length of primary roots was measured.

Callus was induced according to the method reported previously [70]. Briefly, the hypocotyls from 5-day-old dark-grown wild-type and bud2-1 seedlings were excised into 5-mm segments and cultured on GM medium supplemented with the indicated combinations of 2,4-D and zeatin for 30 days at $23{ }^{\circ} \mathrm{C}$ in the dark.

To test expressions of cytokinin or auxin-responsive genes, 12 day-old seedlings of wild-type or the tirlafb2afb3 plants grown on $0.5 \times$ MS plates under constant light were transferred to the same liquid medium supplemented with $10 \mu \mathrm{M}$ of $6-\mathrm{BA}, 10 \mu \mathrm{M}$ of IAA or $0.1 \%$ DMSO. Seedlings were harvested at the time indicated and RNA was isolated for RNA blot or real-time PCR analyses.

\section{Cytokinin measurement}

Extraction and purification of cytokinin were carried out as described previously [78, 79]. Briefly, 2 g aerial parts of 25-day-old plants grown under long-day conditions were homogenized and $200 \mathrm{pmol}$ of each of the following 12 deuterium-labeled standards were immediately added: $\left[{ }^{2} \mathrm{H}_{5}\right] \mathrm{Z},\left[{ }^{2} \mathrm{H}_{5}\right] \mathrm{ZR},\left[{ }^{2} \mathrm{H}_{5}\right] \mathrm{Z} 7 \mathrm{G},\left[{ }^{2} \mathrm{H}_{5}\right] \mathrm{Z} 9 \mathrm{G}$, $\left[{ }^{2} \mathrm{H}_{5}\right] \mathrm{ZOG},\left[{ }^{2} \mathrm{H}_{5}\right] \mathrm{ZROG},\left[{ }^{2} \mathrm{H}_{6}\right] \mathrm{iP},\left[{ }^{2} \mathrm{H}_{6}\right] \mathrm{iPR},\left[{ }^{2} \mathrm{H}_{6}\right] \mathrm{iP} 7 \mathrm{G},\left[{ }^{2} \mathrm{H}_{6}\right] \mathrm{iP} 9 \mathrm{G}$, $\left[{ }^{2} \mathrm{H}_{5}\right] \mathrm{DHZ}$ and $\left[{ }^{2} \mathrm{H}_{5}\right]$ DHZR (Apex Organics, Honiton, Devon, UK). After extraction and SPE purification, cytokinins were analyzed on a liquid chromatography-tandem mass spectrometry system (Finnigan Surveyor LC-LCQ Deca XP MAX, Thermo, San Jose, CA, USA) using positive ion electrospray ionization and quantified as described previously [63].

\section{Generation of the bud2axr1 double mutant}

The double mutant bud2-2axrl-3 was generated from the cross of homozygous bud2-2 with axrl-3 [26], and identified from the $\mathrm{F}_{2}$ progeny grown on soil by comparing with their parental phenotypes and PCR-based characterization.

\section{Acknowledgments}

We thank Dr Ottoline Leyser (University of York, UK) for providing axr 1-3 and Dr Mark Estelle (UC, San Diego, USA) for providing tirlafb2afb3 triple mutant seeds. This work was supported by a grant from the National Natural Science Foundation of China (30830009).

\section{References}

1 Nishimura K, Nakatsu F, Kashiwagi K, Ohno H, Saito T, Igarashi K. Essential role of S-adenosylmethionine decarboxylase in mouse embryonic development. Genes Cells 2002; 7:41-47.

2 Imai A, Akiyama T, Kato T, et al. Spermine is not essential for survival of Arabidopsis. FEBS Lett 2004; 556:148-152.

3 Urano K, Hobo T, Shinozaki K. Arabidopsis ADC genes involved in polyamine biosynthesis are essential for seed development. FEBS Let 2005; 579:1557-1564.

4 Ge C, Cui X, Wang Y, et al. BUD2, encoding an S-adenosylmethionine decarboxylase, is required for Arabidopsis growth and development. Cell Res 2006; 16:446-456.

5 Evans PT, Malmberg RL. Do polyamines have roles in plant development? Annu Rev Plant Physiol Plant Mol Biol 1989; 40:235-269.

6 Hanzawa Y, Takahashi T, Komeda Y. ACL5: an Arabidopsis gene required for internodal elongation after flowering. Plant $J$ 1997; 12:863-874.

7 Watson MB, Emory KK, Piatak RM, Malmberg RL. Arginine 
decarboxylase (polyamine synthesis) mutants of Arabidopsis thaliana exhibit altered root growth. Plant $J$ 1998; 13:231239.

8 Hanzawa Y, Takahashi T, Michael AJ, et al. ACAULIS5, an Arabidopsis gene required for stem elongation, encodes a spermine synthase. EMBO J 2000; 19:4248-4256.

9 Couee I, Hummel I, Sulmon C, Gouesbet G, Amrani A. Involvement of polyamines in root development. Plant Cell Tissue Organ Cult 2004; 76:1-10.

10 Alcázar R, García-Martínez JL, Cuevas JC, Tiburcio AF, Altabella T. Overexpression of ADC2 in Arabidopsis induces dwarfism and late-flowering through GA deficiency. Plant $J$ 2005; 43:425-436.

11 Clay NK, Nelson T. Arabidopsis thickvein mutation affects vein thickness and organ vascularization, and resides in a provascular cell-specific spermine synthase involved in vein definition and in polar auxin transport. Plant Physiol 2005; 138:767-777.

12 Kasinathan V, Wingler A. Effect of reduced arginine decarboxylase activity on salt tolerance and on polyamine formation during salt stress in Arabidopsis thaliana. Physiol Plant 2004; 121:101-107.

13 Kakehi J, Kuwashiro Y, Niitsu M, Takahashi T. Thermospermine is required for stem elongation in Arabidopsis thaliana. Plant Cell Physiol 2008; 49:1342-1349.

14 Thimann KV, Skoog F. On the inhibition of bud development and other functions of growth substance in Vicia faba. Proc $R$ Soc Lond B Biol Sci 1934; 114:317-339.

15 Snow R. On the nature of correlative inhibition. New Phytol 1937; 36:283-300.

16 Morris DR. A new perspective on ornithine decarboxylase regulation: prevention of polyamine toxicity is the overriding theme. J Cell Biochem 1991; 46:102-105.

17 Bangerth F. Dominance among fruits/sinks and the search for a correlative signal. Physiol Plant 1989; 76:608-614.

18 Romano CP, Hein MB, Klee HJ. Inactivation of auxin in tobacco transformed with the indoleacetic acid-lysine synthetase gene of Pseudomonas savastanoi. Genes Dev 1991; 5:438-446.

19 Zhao Y, Christensen SK, Fankhauser C, et al. A role for flavin monooxygenase-like enzymes in auxin biosynthesis. Science 2001; 291:306-309.

20 Bartel B. Auxin biosynthesis. Annu Rev Plant Physiol Plant Mol Biol 1997; 48:51-66.

21 Nakajima E, Yamada K, Kosemura S, Yamamura S, Hasegawa K. Effects of the auxin-inhibiting substances raphanusanin and benzoxazolinone on apical dominance of pea seedlings. Plant Growth Regul 2001; 35:11-15.

22 Chatfield SP, Stirnberg P, Forde BG, Leyser O. The hormonal regulation of axillary bud growth in Arabidopsis. Plant $J$ 2000; 24:159-169.

23 Noh B, Murphy AS, Spalding EP. Multidrug resistance-like genes of Arabidopsis required for auxin transport and auxinmediated development. Plant Cell 2001; 13:2441-2454.

24 Gil P, Dewey E, Friml J, et al. BIG: a calossin-like protein required for polar auxin transport in Arabidopsis. Genes Dev 2001; 15:1985-1997.

25 Dai Y, Wang H, Li B, et al. Increased expression of MAP KINASE KINASE7 causes deficiency in polar auxin transport and leads to plant architectural abnormality in Arabidopsis. Plant Cell 2006; 18:308-320.

26 Leyser HM, Lincoln CA, Timpte C, Lammer D, Turner J, Estelle M. Arabidopsis auxin-resistance gene AXR1 encodes a protein related to ubiquitin-activating enzyme E1. Nature 1993; 364:161-164.

27 Leyser HM, Pickett FB, Dharmasiri S, Estelle M. Mutations in the AXR3 gene of Arabidopsis result in altered auxin response including ectopic expression from the SAUR-AC1 promoter. Plant J 1996; 10:403-413.

28 Booker J, Chatfield S, Leyser O. Auxin acts in xylem-associated or medullary cells to mediate apical dominance. Plant Cell 2003; 15:495-507.

29 Stirnberg P, Chatfield SP, Leyser HM. AXR1 acts after lateral bud formation to inhibit lateral bud growth in Arabidopsis. Plant Physiol 1999; 121:839-847.

30 Morris DA. Transport of exogenous auxin in two-branched dwarf pea seedlings (Pisum sativum L.). Planta 1977; 136:9196.

31 Cline MG. Apical dominance. Bot Rev 1991; 57:318-358.

32 Miyawaki K, Matsumoto-Kitano M, Kakimoto T. Expression of cytokinin biosynthetic isopentenyltransferase genes in Arabidopsis: tissue specificity and regulation by auxin, cytokinin, and nitrate. Plant J 2004; 37:128-138.

33 Tanaka M, Takei K, Kojima M, Sakakibara H, Mori H. Auxin controls local cytokinin biosynthesis in the nodal stem in apical dominance. Plant $J$ 2006; 45:1028-1036.

34 Palni LMS, Burch L, Horgan R. The effect of auxin concentration on cytokinin stability and metabolism. Planta 1988; 174:231-234.

35 Bangerth F. Response of cytokinin concentration in the xylem exudate of bean (Phaseolus vulgaris L.) plants to decapitation and auxin treatment, and relationship to apical dominance. Planta 1994; 194:439-442.

36 Nordstrom A, Tarkowski P, Tarkowska D, et al. Auxin regulation of cytokinin biosynthesis in Arabidopsis thaliana: a factor of potential importance for auxin-cytokinin-regulated development. Proc Natl Acad Sci USA 2004; 101:8039-8044.

37 Stirnberg P, van De Sande K, Leyser HM. MAX1 and MAX2 control shoot lateral branching in Arabidopsis. Development 2002; 129:1131-1141.

38 Sorefan K, Booker J, Haurogne K, et al. MAX4 and RMS1 are orthologous dioxygenase-like genes that regulate shoot branching in Arabidopsis and pea. Genes Dev 2003; 17:14691474.

39 Booker J, Auldridge M, Wills S, McCarty D, Klee H, Leyser O. $\mathrm{MAX} 3 / \mathrm{CCD} 7$ is a carotenoid cleavage dioxygenase required for the synthesis of a novel plant signaling molecule. Curr Biol 2004; 14:1232-1238.

40 Booker J, Sieberer T, Wright W, et al. MAX1 encodes a cytochrome P450 family member that acts downstream of MAX3/4 to produce a carotenoid-derived branch-inhibiting hormone. Dev Cell 2005; 8:443-449.

41 Beveridge CA, Ross JJ, Murfet IC. Branching mutant $r m s-2$ in Pisum sativum (Grafting studies and endogenous indole-3acetic acid levels). Plant Physiol 1994; 104:953-959.

42 Beveridge CA, Ross JJ, Murfet IC. Branching in pea (action of genes Rms 3 and Rms4). Plant Physiol 1996; 110:859-865.

43 Beveridge CA, Symons GM, Turnbull CG. Auxin inhibition 
of decapitation-induced branching is dependent on grafttransmissible signals regulated by genes Rms 1 and $R m s 2$. Plant Physiol 2000; 123:689-698.

44 Napoli C. Highly branched phenotype of the Petunia dad1-1 mutant is reversed by grafting. Plant Physiol 1996; 111:2737.

45 Simons JL, Napoli CA, Janssen BJ, Plummer KM, Snowden KC. Analysis of the DECREASED APICAL DOMINANCE genes of petunia in the control of axillary branching. Plant Physiol 2007; 143:697-706.

46 Ishikawa S, Maekawa M, Arite T, Onishi K, Takamure I, Kyozuka J. Suppression of tiller bud activity in tillering dwarf mutants of rice. Plant Cell Physiol 2005; 46:79-86.

47 Arite T, Iwata $\mathrm{H}$, Ohshima K, et al. DWARF10, an RMS1/ $M A X 4 / D A D 1$ ortholog, controls lateral bud outgrowth in rice. Plant J 2007; 51:1019-1029.

48 Zou J, Zhang S, Zhang W, et al. The rice HIGH-TILLERING $D W A R F 1$ encoding an ortholog of Arabidopsis MAX3 is required for negative regulation of the outgrowth of axillary buds. Plant J 2006; 48:687-698.

49 Lin H, Wang R, Qian Q, et al. DWARF27, an iron-containing protein required for the biosynthesis of strigolactones, regulates rice tiller bud outgrowth. Plant Cell 2009; 21:15121525.

50 Gao Z, Qian Q, Liu X, et al. Dwarf 88, a novel putative esterase gene affecting architecture of rice plant. Plant Mol Biol 2009; 71:265-276.

51 Arite T, Umehara M, Ishikawa S, et al. d14, a strigolactoneinsensitive mutant of rice, shows an accelerated outgrowth of tillers. Plant Cell Physiol 2009; 50:1416-1424.

52 Umehara M, Hanada A, Yoshida S, et al. Inhibition of shoot branching by new terpenoid plant hormones. Nature 2008; 455: 195-200.

53 Gomez-Roldan V, Fermas S, Brewer PB, et al. Strigolactone inhibition of shoot branching. Nature 2008; 455:189-194.

54 Beveridge CA, Symono GM, Murfet IC, Ross JJ, Rameau C. The rms 1 mutant of pea has elevated indole-3-acetic acid levels and reduced root-sap zeatin riboside content but increased branching controlled by graft-transmissible signal(s). Plant Physiol 1997; 115:1251-1258.

55 Morris SE, Turnbull CG, Murfet IC, Beveridge CA. Mutational analysis of branching in pea. Evidence that Rms 1 and Rms5 regulate the same novel signal. Plant Physiol 2001; 126:1205-1213.

56 Turnbull CG, Booker JP, Leyser HM. Micrografting techniques for testing long-distance signalling in Arabidopsis. Plant J 2002; 32:255-262.

57 Shimizu S, Mori H. Analysis of cycles of dormancy and growth in pea axillary buds based on mRNA accumulation patterns of cell cycle-related genes. Plant Cell Physiol 1998; 39:255-262.

58 Geuns JM, Smets R, Struyf T, Prinsen E, Valcke R, Van Onckelen H. Apical dominance in Pssu-ipt-transformed tobacco. Phytochemistry 2001; 58:911-921.

59 Gray WM, Ostin A, Sandberg G, Romano CP, Estelle M. High temperature promotes auxin-mediated hypocotyl elongation in Arabidopsis. Proc Natl Acad Sci USA 1998; 95:7197-7202.

60 Lincoln C, Britton JH, Estelle M. Growth and development of the axrl mutants of Arabidopsis. Plant Cell 1990; 2:1071-1071
1080.

61 Abel S, Nguyen MD, Theologis A. The PS-IAA4/5-like family of early auxin-inducible mRNAs in Arabidopsis thaliana. J Mol Biol 1995; 251:533-549.

62 Dharmasiri N, Dharmasiri S, Weijers D, et al. Plant development is regulated by a family of auxin receptor F-box proteins. Dev Cell 2005; 9:109-119.

63 Nakagawa H, Jiang CJ, Sakakibara H, et al. Overexpression of a petunia zinc-finger gene alters cytokinin metabolism and plant forms. Plant $J 2005$; 41:512-523.

64 Tantikanjana T, Yong JW, Letham DS, et al. Control of axillary bud initiation and shoot architecture in Arabidopsis through the SUPERSHOOT gene. Genes Dev 2001; 15:15771588.

65 Helliwell CA, Chin-Atkins AN, Wilson IW, Chapple R, Dennis ES, Chaudhury A. The Arabidopsis AMP1 gene encodes a putative glutamate carboxypeptidase. Plant Cell 2001; 13:2115-2125.

66 Brandstatter I, Kieber JJ. Two genes with similarity to bacterial response regulators are rapidly and specifically induced by cytokinin in Arabidopsis. Plant Cell 1998; 10:1009-1019.

67 Kiba T, Yamada H, Sato S, et al. The type-A response regulator, $A R R 15$, acts as a negative regulator in the cytokininmediated signal transduction in Arabidopsis thaliana. Plant Cell Physiol 2003; 44:868-874.

68 Kiba T, Yamada H, Mizuno T. Characterization of the ARR15 and ARR16 response regulators with special reference to the cytokinin signaling pathway mediated by the AHK4 histidine kinase in roots of Arabidopsis thaliana. Plant cell Physiol 2002; 43:1059-1066.

69 D'Agostino IB, Deruere J, Kieber JJ. Characterization of the response of the Arabidopsis response regulator gene family to cytokinin. Plant Physiol 2000; 124:1706-1717.

70 Kubo M, Kakimoto T. The cytokinin-hypersensitive genes of Arabidopsis negatively regulate the cytokinin-signaling pathway for cell division and chloroplast development. Plant $J$ 2000; 23:385-394.

71 Imai A, Matsuyama T, Hanzawa Y, et al. Spermidine synthase genes are essential for survival of Arabidopsis. Plant Physiol 2004; 135:1565-1573.

72 Soyka S, Heyer AG. Arabidopsis knockout mutation of ADC2 gene reveals inducibility by osmotic stress. FEBS Lett 1999; 458:219-223.

73 Wilmoth JC, Wang S, Tiwari SB, et al. NPH4/ARF7 and $A R F 19$ promote leaf expansion and auxin-induced lateral root formation. Plant J 2005; 43:118-130.

74 Harrar Y, Bellec Y, Bellini C, Faure JD. Hormonal control of cell proliferation requires PASTICCINO genes. Plant Physiol 2003; 132:1217-1227.

75 Cona A, Cenci F, Cervelli M, et al. Polyamine oxidase, a hydrogen peroxide-producing enzyme, is up-regulated by light and down-regulated by auxin in the outer tissues of the maize mesocotyl. Plant Physiol 2003; 131:803-813.

76 Mou Z, He Y, Dai Y, Liu X, Li J. Deficiency in fatty acid synthase leads to premature cell death and dramatic alterations in plant morphology. Plant Cell 2000; 12:405-418.

$77 \mathrm{Hu}$ Y, Bao F, Li J. Promotive effect of brassinosteroids on cell division involves a distinct $\mathrm{CycD} 3$-induction pathway in $\mathrm{Ara}$ bidopsis. Plant J 2000; 24:693-601. 
78 Dobrev PI, Kaminek M. Fast and efficient separation of cytokinins from auxin and abscisic acid and their purification using mixed-mode solid-phase extraction. J Chromatogr A 2002; 950:21-29.
79 Hoyerova K, Gaudinova A, Malbeck J, et al. Efficiency of different methods of extraction and purification of cytokinins. Phytochemistry 2006; 67:1151-1159.

(Supplementary information is linked to the online version of the paper on the Cell Research website.) 